\title{
La formación inicial de los educadores sociales en el ámbito de servicios sociales
}

\section{Initial training of social educators in the field of social services}

\author{
Laura Varela Crespo \\ Universidad de Santiago de Compostela
}

\begin{abstract}
Resumen
El trabajo se centra en el estudio de las guías docentes de los Grados en Educación Social de las universidades españolas, con el objetivo de valorar la capacitación que reciben los estudiantes en materia de servicios sociales. Aun siendo un ámbito esencial de inserción profesional de estos agentes educativos, se aprecian déficits formativos en torno a las posibilidades de obtención de un conocimiento profundo sobre la organización y estructura de los servicios sociales, el marco normativo, las funciones profesionales a llevar a cabo en este ámbito de acción profesional, etc., al finalizar los estudios de Grado.

Palabras clave: formación inicial, educación social, servicio social.
\end{abstract}

\section{Abstract}

The paper focuses on the study of teaching guides of degrees in Social Education of the Spanish universities, with the objective of evaluating the training that students receive in the area of social services. Even though it is an essential field of professional insertion of these educational agents, there are formative deficits regarding the possibilities of obtaining a deep knowledge about the organization and structure of social services, the normative framework, the professional functions to be carried out in this field, etc., at the end of the studies of Degree.

Keywords: initial training, community education, social service.

\section{Los servicios sociales como escenario de actuación profesional de la Educación Social}

Considerando la Educación Social como una práctica socioeducativa orientada a la incorporación de los sujetos de la educación a la diversidad de redes sociales y a la promoción social y cultural de la ciudadanía (ASEDES, 2004), resulta fundamental traer al centro del debate pedagógico los significados que adopta en el marco de los servicios sociales, así como la formación que las educadoras y los educadores sociales reciben en este ámbito de intervención. En palabras de Moyano (2012, p. 43), la emergencia de la educación social en el campo de los servicios sociales ha abierto la "oportunidad de añadir un plus que distancie los elementos estigmatizantes y dirija sus objetivos hacia el horizonte de la promoción cultural de los individuos que atiende". Oportunidad que ha de ser aprovechada en un tiempo dominado por las políticas de austeridad, donde la Educación Social corre el riesgo de desprofesionalizarse y de ver limitada su tarea a la mera ejecución de prácticas compensadoras.

En el contexto español, se han producido -aun con importantes limitaciones en inversión y coberturaavances destacados desde la primera generación de leyes de servicios sociales promulgadas en la década de los 80 .
Estas referencias normativas integraban las prestaciones básicas del sistema (información y orientación; ayuda a domicilio y otros apoyos a la unidad de convivencia; alojamiento alternativo; prevención e inserción social; cooperación social y fomento de la solidaridad) evolucionando hasta llegar a las leyes actuales aprobadas en la última década del siglo XXI en diversas Comunidades Autónomas, presentando un mayor desarrollo respecto a la descentralización, universalización y garantía de acceso.

Cabe destacar que en la delimitación conceptual de estos servicios se distingue una doble perspectiva, amplia y restringida. La primera incluye el conjunto de medidas, prestaciones y actividades que tienen por objetivo mejorar la calidad de vida (sanidad, educación, vivienda, políticas de ocupación, etc.), mientras que la segunda predominante en el contexto español- entiende los servicios sociales como el conjunto coordinado de programas, recursos, equipamientos y prestaciones que garantizan a las personas su derecho a obtener respuestas ante situaciones de necesidad, en los ámbitos de información y orientación, convivencia, promoción e integración, participación y solidaridad social, etc., a través de intervenciones profesionales en un territorio determinado. Desde esta perspectiva restringida, los servicios sociales se estructuran en dos niveles: comunitarios (también denominados de atención primaria) y especializados; constituyendo los primeros el nivel de atención más próximo y el canal normal de acceso, y los segundos, referidos a situaciones de gran complejidad y que exigen una alta especialización técnica.

Se ha de tener en cuenta que -más allá de los aspectos organizativos y de gestión- en el buen funcionamiento de los servicios sociales incide ineludiblemente la dimensión humana, dado que su quehacer cotidiano se genera a partir de intercambios que presentan una "alta intensidad relacional" entre el profesional y la persona/grupo/comunidad con la que trabaja (García Roca, 2006). Son servicios que no pueden ser ni mercantilizados, ni administrados y que dependen esencialmente de las relaciones que se establecen por parte de los sujetos que intervienen. De ahí la relevancia de la acción socioeducativa y la necesidad de las profesiones educadoras en este ámbito.

Las funciones que llevan a cabo los educadores y educadoras sociales en los servicios sociales se centran en: el diseño y ejecución de programas; las actividades de prevención e inserción social; el acompañamiento familiar; la intervención socioeducativa; el desarrollo de 
programas para colectivos con necesidades específicas; la intervención comunitaria; etc. Un conjunto de funciones que deben tener su reflejo en la formación inicial mediante contenidos específicos que aborden la estructura organizativa y funcional de los servicios sociales, las áreas de actuación, las principales prestaciones (técnicas, materiales, etc.) y las funciones/competencias de los educadores y educadoras sociales en este escenario de intervención.

\section{Método}

El trabajo tiene por objetivo analizar la formación de los educadores y educadoras sociales en el ámbito de los servicios sociales, al constituir uno de los principales escenarios de inserción laboral de esta figura profesional en el contexto español. Si bien la capacitación en torno a los colectivos a los que se presta atención desde el nivel especializado (infancia, juventud, mayores, etc.) es abordada con amplitud en los Grados en Educación Social, partimos de la hipótesis de que la formación respecto al sistema público de servicios sociales especialmente a su estructura y organización, funciones profesionales, marco normativo, etc.- resulta insuficiente.

Con este propósito se ha realizado una revisión documental de las guías docentes del curso 2016/2017 de los Planes de Estudio del Grado en Educación Social de las universidades españolas que ofertan esta titulación, que conforman un total de 34; siendo 29 públicas y 5 privadas $^{2}$ (tabla 1). En concreto, se ha analizado el apartado de "contenidos" de las guías docentes de las materias que abordan la temática de los servicios sociales como eje central.

Tabla 1.

Universidades españolas que ofertan el Grado en Educación Social (curso 2016-2017)

\begin{tabular}{|c|c|c|c|}
\hline $\begin{array}{l}\text { Comunidad } \\
\text { autónoma }\end{array}$ & Universidad & $\begin{array}{l}\text { Comunidad } \\
\text { autónoma }\end{array}$ & Universidad \\
\hline Andalucía & $\begin{array}{l}\text { UAL } \\
\text { Almería } \\
\text { UCO } \\
\text { Córdoba } \\
\text { UG } \\
\text { Granada } \\
\text { UHU } \\
\text { Huelva } \\
\text { UJA Jaén } \\
\text { UMA } \\
\text { Málaga } \\
\text { UPO Pablo } \\
\text { de Olavide }\end{array}$ & Extremadura & $\begin{array}{l}\text { UEX } \\
\text { Extremadura }\end{array}$ \\
\hline Asturias & $\begin{array}{l}\text { UO } \\
\text { Oviedo }\end{array}$ & Galicia & $\begin{array}{l}\text { UDC A } \\
\text { Coruña } \\
\text { USC } \\
\text { Santiago } \\
\text { Compostela }\end{array}$ \\
\hline \multicolumn{4}{|c|}{$\begin{array}{l}{ }^{1} \text { En la Universidad de Granada las guías docentes revisadas se } \\
\text { corresponden con el curso 2015/2016, al no tener acceso en la página } \\
\text { web de dicha universidad a las correspondientes al curso 2016/2017. } \\
2 \text { Las Universidades privadas que ofertan el Grado en Educación Social } \\
\text { son: Oberta de Catalunya, Ramón Llull, Vic, Católica de Valencia y } \\
\text { Deusto. Las restantes son de carácter público. }\end{array}$} \\
\hline
\end{tabular}

\begin{tabular}{|c|c|c|c|}
\hline & & & UVigo Vigo \\
\hline Canarias & $\begin{array}{l}\text { ULPGC } \\
\text { Palmas de G. } \\
\text { Canaria }\end{array}$ & $\begin{array}{l}\text { Islas } \\
\text { Baleares }\end{array}$ & $\begin{array}{l}\text { UIB Islas } \\
\text { Baleares }\end{array}$ \\
\hline $\begin{array}{l}\text { Castilla La } \\
\text { Mancha }\end{array}$ & $\begin{array}{l}\text { UCLM } \\
\text { Castilla la } \\
\text { Mancha }\end{array}$ & Madrid & $\begin{array}{l}\text { UAH Alcalá } \\
\text { de Henares } \\
\text { UCM } \\
\text { Complutense } \\
\text { Madrid }\end{array}$ \\
\hline $\begin{array}{l}\text { Castilla y } \\
\text { León }\end{array}$ & $\begin{array}{l}\text { UBU } \\
\text { Burgos } \\
\text { ULE León } \\
\text { USAL } \\
\text { Salamanca } \\
\text { UVa } \\
\text { Valladolid }\end{array}$ & Murcia & UM Murcia \\
\hline Cataluña & $\begin{array}{l}\text { UAB } \\
\text { Autónoma } \\
\text { Barcelona } \\
\text { UB } \\
\text { Barcelona } \\
\text { UOC } \\
\text { Oberta de } \\
\text { Catalunya } \\
\text { URL } \\
\text { Ramón Llull } \\
\text { UVic Vic } \\
\text { UdG Girona } \\
\text { UdL } \\
\text { Lleida } \\
\text { URV } \\
\text { Rovira i } \\
\text { Virgili }\end{array}$ & País Vasco & $\begin{array}{l}\text { UD } \\
\text { Deusto } \\
\text { UPV País } \\
\text { Vasco }\end{array}$ \\
\hline $\begin{array}{l}\text { Comunidad } \\
\text { Valenciana }\end{array}$ & $\begin{array}{l}\text { UCV } \\
\text { Católica de } \\
\text { Valencia } \\
\text { UV } \\
\text { Valencia }\end{array}$ & Estado & UNED \\
\hline
\end{tabular}

Para el análisis del contenido de las materias específicas sobre servicios sociales se hizo uso del programa ATLAS.ti, en base al siguiente sistema de codificación elaborado de modo inductivo a partir de la lectura de los epígrafes de contenido de las guías docentes de las diferentes universidades.

Tabla 2.

Sistema de codificación utilizado para el análisis de las guías docentes

\begin{tabular}{ll}
\hline \multicolumn{1}{c}{ Código } & \multicolumn{1}{c}{ Definición } \\
\hline Bienestar social & $\begin{array}{l}\text { Concepto, características, } \\
\text { dimensiones }\end{array}$ \\
\hline Política social & Concepto, modelos \\
\hline $\begin{array}{l}\text { Colectivos/personas } \\
\text { destinatarias }\end{array}$ & $\begin{array}{l}\text { Colectivos destinatarios de los } \\
\text { servicios sociales }\end{array}$ \\
\hline $\begin{array}{l}\text { Estado/sociedad de } \\
\text { bienestar/crisis Estado } \\
\text { de bienestar }\end{array}$ & $\begin{array}{l}\text { Concepto, características, } \\
\text { evolución, crisis de Estado de } \\
\text { bienestar Sociedad del bienestar }\end{array}$ \\
\hline Exclusión y pobreza & $\begin{array}{l}\text { Concepto. Proceso y } \\
\text { multidimensionalidad }\end{array}$ \\
\hline Ética y servicios sociales & $\begin{array}{l}\text { Ética aplicada a los servicios } \\
\text { sociales }\end{array}$ \\
\hline
\end{tabular}




\begin{tabular}{ll}
\hline $\begin{array}{l}\text { Funciones educador } \\
\text { social }\end{array}$ & $\begin{array}{l}\text { Funciones y competencias en el } \\
\text { marco de equipos } \\
\text { interdisciplinares. }\end{array}$ \\
\hline $\begin{array}{l}\text { Acción/intervención } \\
\text { socioeducativa }\end{array}$ & $\begin{array}{l}\text { Concepto, modelos de } \\
\text { intervención, } \\
\text { instrumentos/técnicas }\end{array}$ \\
\hline $\begin{array}{l}\text { Marco } \\
\text { normativo-declaraciones } \\
\text { internacionales }\end{array}$ & $\begin{array}{l}\text { Legislación internacional, } \\
\text { autonómica y local de servicios } \\
\text { sociales }\end{array}$ \\
\hline Necesidades sociales & $\begin{array}{l}\text { Necesidades, demandas, derechos. } \\
\text { Tipología de necesidades. } \\
\text { Satisfactores/recursos }\end{array}$ \\
\hline $\begin{array}{l}\text { Planificación y } \\
\text { evaluación }\end{array}$ & $\begin{array}{l}\text { Planificación y evaluación en } \\
\text { servicios sociales }\end{array}$ \\
\hline Prestaciones & $\begin{array}{l}\text { Prestaciones, programas y } \\
\text { servicios }\end{array}$ \\
\hline $\begin{array}{l}\text { Servicios sociales: } \\
\text { concepto }\end{array}$ & $\begin{array}{l}\text { Definición, componentes, } \\
\text { naturaleza del sistema público de } \\
\text { servicios sociales }\end{array}$ \\
\hline $\begin{array}{l}\text { Servicios sociales: } \\
\text { estructura y } \\
\text { organización }\end{array}$ & $\begin{array}{l}\text { Estructura y organización del } \\
\text { sistema público de servicios } \\
\text { sociales }\end{array}$ \\
\hline $\begin{array}{l}\text { Servicios sociales: } \\
\text { evolución histórica }\end{array}$ & $\begin{array}{l}\text { Evolución histórica y desarrollo de } \\
\text { los servicios sociales }\end{array}$ \\
\hline $\begin{array}{l}\text { Ámbitos Servicios } \\
\text { sociales especializados }\end{array}$ & $\begin{array}{l}\text { Ambitos de actuación de los } \\
\text { servicios sociales especializados }\end{array}$ \\
\hline $\begin{array}{l}\text { Trabajo } \\
\text { comunitario/iniciativa } \\
\text { ciudadana }\end{array}$ & $\begin{array}{l}\text { Trabajo comunitario, dinamización } \\
\text { del territorio, participación e } \\
\text { iniciativa ciudadana }\end{array}$ \\
\hline Trabajo en red & $\begin{array}{l}\text { Trabajo en red, trabajo en equipo y } \\
\text { coordinación }\end{array}$ \\
\hline
\end{tabular}

Además, en el caso de aquellas universidades que no cuentan con una materia específica dedicada a los servicios sociales, se realizó una revisión de la guía docente de la materia "Pedagogía Social", dado que entre los ámbitos de intervención del educador/a social (contenido específico de esta materia) se encuentra el de los servicios sociales. Asimismo, se indagó en los contenidos de la asignatura "Contextos y ámbitos en Educación Social" (que recibe diferentes denominaciones según las universidades) referida a los escenarios de inserción profesional, entre los que se sitúa la temática objeto de estudio. Complementariamente, se analizaron las guías docentes de otras materias en las cuales los servicios sociales podrían tener cabida y que en su denominación contuviesen alguna de las siguientes palabras clave: política(s) social(es); estado del bienestar; políticas de bienestar social; legislación social.

\section{Resultados}

Tras el análisis de las guías docentes de las universidades españolas que ofertan el Grado en Educación Social, cabe destacar que el abordaje de los servicios sociales como asignatura específica resulta insuficiente dado que del total de las 34 universidades que ofertan esta titulación, solamente se contempla como materia propia en 14 planes de estudio; es decir, en el $41 \%$ de las instituciones de educación superior. No obstante, en once universidades que cuentan con esta formación, predomina el carácter obligatorio o de formación básica de la asignatura ${ }^{3}$ lo que supone que todo el alumnado matriculado debe cursar de forma ineludible este tipo de contenidos, teniendo carácter optativo en tres de las universidades analizadas (tabla 3). $\mathrm{Si}$ bien en las instituciones de educación superior en las que se incorpora este tipo de formación constituye un contenido central, resulta preocupante que en el $56 \%$ de los planes de estudio no se ofrezca capacitación específica en torno a este ámbito de trabajo.

Tabla 3.

Asignaturas especificas sobre los servicios sociales en el Grado en Educación Social (curso 2016/2017)

\begin{tabular}{|c|c|c|c|c|}
\hline \multirow{2}{*}{ Universidad } & \multirow{2}{*}{ Materias } & \multicolumn{3}{|c|}{ Tipo $^{4}$} \\
\hline & & $\mathrm{OB}$ & FB & $\mathrm{OP}$ \\
\hline UHU & $\begin{array}{l}\text { Servicios } \\
\text { sociales }\end{array}$ & $\mathrm{x}$ & & \\
\hline UJA & $\begin{array}{l}\text { Las políticas del } \\
\text { bienestar. } \\
\text { Sistemas de } \\
\text { protección social y } \\
\text { servicios sociales }\end{array}$ & & $\mathrm{x}$ & \\
\hline UPO & $\begin{array}{l}\text { Servicios } \\
\text { sociales }\end{array}$ & $\mathrm{x}$ & & \\
\hline & $\begin{array}{l}\text { Servicios } \\
\text { sociales }\end{array}$ & & $\mathrm{x}$ & \\
\hline UCLM & $\begin{array}{c}\text { El educador } \\
\text { social en los } \\
\text { servicios sociales } \\
\end{array}$ & & $\mathrm{x}$ & \\
\hline USAL & $\begin{array}{l}\text { Servicios } \\
\text { sociales }\end{array}$ & $\mathrm{x}$ & & \\
\hline UB & $\begin{array}{c}\text { Acción } \\
\text { socioeducativa en } \\
\text { los servicios } \\
\text { sociales } \\
\end{array}$ & & & $\mathrm{x}$ \\
\hline UOC & $\begin{array}{l}\text { Servicios } \\
\text { sociales }\end{array}$ & & $\mathrm{x}$ & \\
\hline URL & $\begin{array}{l}\text { Servicios } \\
\text { sociales }\end{array}$ & $\mathrm{x}$ & & \\
\hline UVic & $\begin{array}{c}\text { Políticas y } \\
\text { servicios sociales }\end{array}$ & $\mathrm{x}$ & & \\
\hline $\mathrm{UdG}$ & $\begin{array}{c}\text { El educador } \\
\text { social en el ámbito } \\
\text { de la atención } \\
\text { primaria }\end{array}$ & & & $\mathrm{x}$ \\
\hline UDC & $\begin{array}{c}\text { Servicios } \\
\text { sociales y } \\
\text { bienestar social } \\
\end{array}$ & $\mathrm{x}$ & & \\
\hline USC & $\begin{array}{c}\text { Educación } \\
\text { social, servicios } \\
\text { sociales e } \\
\text { iniciativa } \\
\text { ciudadana } \\
\end{array}$ & $\mathrm{x}$ & & \\
\hline UVigo & $\begin{array}{c}\text { Educación } \\
\text { social en los } \\
\text { servicios sociales }\end{array}$ & & & $\mathrm{x}$ \\
\hline UD & $\begin{array}{c}\text { Servicios } \\
\text { sociales en el } \\
\text { Estado de } \\
\text { bienestar }\end{array}$ & $\mathrm{x}$ & & \\
\hline
\end{tabular}

${ }^{3}$ La Universidad de Castilla La Mancha oferta dos asignaturas específicas en el ámbito de servicios sociales.

4 Tipología de asignaturas: OB (obligatoria); FB (formación básica); OP (optativa). 


\begin{tabular}{ccccc}
\hline UV & $\begin{array}{c}\text { Administración } \\
\text { social y sistemas } \\
\text { de bienestar }\end{array}$ & $\mathrm{x}$ & & \\
\hline Total: 15 & Total: 16 & 9 & 4 & 3 \\
\hline
\end{tabular}

Tabla 4.

Contenidos de las guías docentes de las materias específicas de servicios sociales (curso 2016/2017)

\begin{tabular}{lc}
\hline \multicolumn{1}{c}{ Código } & $\begin{array}{c}\text { Número de veces } \\
\text { citado en las guías } \\
\text { docentes }\end{array}$ \\
\hline Bienestar social & 3 \\
\hline Política social & 5 \\
\hline Colectivos a los que se dirige & 5 \\
\hline $\begin{array}{l}\text { Estado/sociedad de bienestar/crisis } \\
\text { Estado de bienestar }\end{array}$ & 9 \\
\hline Exclusión y pobreza & 3 \\
\hline Ética y servicios sociales & 2 \\
\hline Funciones educador social & 3 \\
\hline $\begin{array}{l}\text { Acción/intervención } \\
\text { socioeducativa }\end{array}$ & 9 \\
\hline Marco normativo & 11 \\
\hline Necesidades sociales & 8 \\
\hline Planificación y evaluación & 2 \\
\hline Prestaciones & 5 \\
\hline Servicios sociales: concepto & 11 \\
\hline $\begin{array}{l}\text { Servicios sociales: estructura y } \\
\text { organización }\end{array}$ & 15 \\
\hline $\begin{array}{l}\text { Servicios sociales: evolución } \\
\text { histórica }\end{array}$ & 7 \\
\hline $\begin{array}{l}\text { Trabajo comunitario/iniciativa } \\
\text { ciudadana }\end{array}$ & 3 \\
\hline Trabajo en red & 1 \\
\hline
\end{tabular}

En las universidades que cuentan con una materia específica para el estudio de esta temática (tabla 4), los contenidos se centran -por orden de frecuencia de aparición-en los siguientes descriptores:

- Concepto, estructura y organización de los servicios sociales

- Marco normativo-legal

- Estado del bienestar: concepto, características, evolución

- Acción e intervención socioeducativa en servicios sociales

- Necesidades sociales

- Evolución histórica de los servicios sociales

Por el contrario, los contenidos a los que se les presta una menor atención en el marco de estas asignaturas son: trabajo en red; planificación y evaluación; trabajo comunitario/iniciativa ciudadana; ética y servicios sociales; funciones del educador/a social; y bienestar social.

En cuanto a las universidades que no incluyen una materia específica en sus planes de estudios (19), solamente en 4 de ellas los servicios sociales conforman un tema o subtema del programa (tabla 5); quedando de este modo relegada a un segundo plano esta temática de estudio en la formación inicial de las educadoras y los educadores sociales.
Tabla 5.

Otras asignaturas que incluyen temas/subtemas especificos de servicios sociales (curso 2016/2017)

\begin{tabular}{|c|c|c|}
\hline Universidad & Asignatura & Contenido \\
\hline Almería & $\begin{array}{l}\text { Políticas sociales y } \\
\text { Estado del } \\
\text { Bienestar } \\
\text { ( } 1^{\circ} \text { curso, básica, } 6 \\
\text { créditos) }\end{array}$ & $\begin{array}{l}\text { Aspectos } \\
\text { introductorios y } \\
\text { conceptuales. El } \\
\text { Estado de Bienestar } \\
\text { Social: Sistemas } \\
\text { Públicos de } \\
\text { Bienestar. Políticas } \\
\text { sociales e Ideología. } \\
\text { Unión Europea y } \\
\text { España: } \\
\text { Evolución de las } \\
\text { políticas sociales. } \\
\text { Legislación en } \\
\text { materia de Servicios } \\
\text { Sociales de la } \\
\text { Comunidad } \\
\text { Autónoma de } \\
\text { Andalucía. Modelos } \\
\text { de organización de } \\
\text { los Servicios Sociales } \\
\text { Municipales aspectos } \\
\text { diferenciales de las } \\
\text { grandes ciudades y } \\
\text { los pequeños } \\
\text { municipios. El futuro } \\
\text { del estado del } \\
\text { bienestar y de las } \\
\text { políticas sociales }\end{array}$ \\
\hline Baleares & $\begin{array}{l}\text { Intervención } \\
\text { socioeducativa } \\
\text { sobre los menores } \\
\text { con problemas de } \\
\text { inadaptación } \\
\text { social }\left(2^{\circ} \text { curso, }\right. \\
\text { obligatoria, } 6 \\
\text { créditos) }\end{array}$ & $\begin{array}{l}\text { Unidad didáctica } 8 . \\
\text { Los servicios sociales } \\
\text { en el marco de los } \\
\text { Servicios de } \\
\text { Bienestar }\end{array}$ \\
\hline & $\begin{array}{l}\text { Pedagogía social } \\
\left(2^{\circ} \text { curso, básica, } 6\right. \\
\text { créditos) }\end{array}$ & $\begin{array}{l}\text { Tema 9. Los servicios } \\
\text { sociales: historia, } \\
\text { conceptos y campos } \\
\text { de } \\
\text { actuación }\end{array}$ \\
\hline Burgos & $\begin{array}{l}\text { Marco jurídico de } \\
\text { la Educación } \\
\text { social y políticas } \\
\text { sociales ( } 1^{\circ} \text { curso, } \\
\text { básica, } 6 \text { créditos) }\end{array}$ & $\begin{array}{l}\text { Tema 2. Legislación } \\
\text { y Educación social } \\
\text { 2.1. Cuestiones } \\
\text { elementales sobre la } \\
\text { Legislación de } \\
\text { servicios sociales y } \\
\text { acción social } \\
\text { 2.2. Aspectos } \\
\text { generales sobre la } \\
\text { normativa relativa al } \\
\text { voluntariado }\end{array}$ \\
\hline Oviedo & $\begin{array}{l}\text { Política social y } \\
\text { gestión de } \\
\text { instituciones } \\
\left(3^{\circ} \text { curso, }\right. \\
\text { obligatoria, } 6 \\
\text { créditos) }\end{array}$ & $\begin{array}{l}\text { Tema 4. Los } \\
\text { Servicios Sociales en } \\
\text { las Administraciones } \\
\text { Públicas y la } \\
\text { Economía Social. } \\
\text { 4.1 Servicios Sociales } \\
\text { en las } \\
\text { Administraciones } \\
\text { Públicas: } \\
\text { 4.1.1. Política Social } \\
\text { Estatal. }\end{array}$ \\
\hline
\end{tabular}




\begin{tabular}{lll}
\hline & & 4.1 .2$. Política Social \\
& & Autonómica. \\
& 4.1 .3 . Política Social \\
& Municipal. \\
\hline \multirow{3}{*}{ Lleida } & Contextos y & Tema 1. Los servicios \\
& ámbitos de la & sociales: marco legal, \\
& educación social II & características de la \\
& $\left(1^{\circ}\right.$ curso, & población, red de \\
& obligatoria, 6 & recursos y acción \\
créditos $)$ & socioeducativa \\
\hline
\end{tabular}

Una situación que resulta preocupante si se tiene en cuenta que la formación inicial es la única oportunidad compartida de manera general por el conjunto del alumnado para aproximarse a los servicios sociales con una perspectiva universalista y de derechos de la ciudadanía, más allá de la intervención con colectivos específicos (mujer, mayores, infancia, etc.) incluidos en otras materias del Grado. En este sentido, hay que destacar que de las 19 universidades que no cuentan con ninguna materia específica, 14 de ellas no incluyen en las guías docentes de ninguna asignatura el contenido de servicios sociales (Córdoba, Granada, Málaga, Las Palmas de Gran Canaria, León, Valladolid, Autónoma de Barcelona, Rovira i Virgili, UNED, Católica de Valencia, Extremadura, Alcalá, Complutense de Madrid, Murcia, País Vasco). Además, la perspectiva aplicada de esta asignatura mediante la aproximación a la realidad social a través del estudio de casos solamente es incluida explícitamente en las guías docentes de las Universidades de Santiago de Compostela y Oberta de Catalunya.

\section{Conclusiones: algunos desafíos en la formación inicial}

La incorporación de los educadores y educadoras sociales a los servicios sociales de los ayuntamientos españoles, como administración más próxima a la ciudadanía, se ha ido produciendo de modo progresivo a lo largo de los últimos 25 años, constituyendo a día de hoy un perfil profesional fundamental en los equipos interprofesionales que integran los servicios sociales municipales (junto con trabajadores/as sociales, psicólogos/as, etc.). No obstante, siendo un ámbito importante de inserción profesional de estos agentes educativos, se aprecian déficits formativos en torno a las posibilidades de obtención de un conocimiento profundo sobre la organización y estructura, el marco normativo, las prestaciones, programas y equipamientos que forman parte de la red de estos servicios, y otros aspectos que resultan fundamentales para el desempeño profesional como es el estudio del papel del educador/a social y de las funciones específicas a llevar a cabo en este ámbito de actuación.

Siendo cierto que la especialización del profesional se realizará en el propio servicio donde trabaje, en función de los programas o proyectos en los que se centre su labor, y mediante la participación en iniciativas de formación permanente, la capacitación universitaria ha de ofrecer a cualquier futuro educador o educadora social un conjunto de conocimientos, habilidades y actitudes que le posibiliten intervenir educativamente en aquellas situaciones que requieren de su mediación; destacando el estudio de la intervención socioeducativa (modelos, metodologías, etc.), las funciones a desempeñar por el educador/a social y el trabajo comunitario indispensable para poder desarrollar actuaciones de carácter preventivo y de fomento de la participación superando el asistencialismo.

Sin embargo, los resultados obtenidos se distancian de esta pretensión al constatarse la existencia de una gran disparidad y de un notable vacío formativo en la titulación de Educación Social en torno a este escenario de intervención, resultando insuficiente que 15 universidades de las 34 que en España ofertan el título de Grado incorporen una materia específica, manteniendo en las restantes la temática un carácter residual (5) o inexistente (14). Una situación en la que insiste Ramírez Navarro (Morán y Varela, 2017), presidente de la Asociación de directoras y gerentes de servicios sociales, al afirmar que en la formación relacionada con los servicios sociales hay un gran déficit, en el sentido de que un educador/a debe ser conocedor del sistema público de protección social en el que va a trabajar. Para este autor, resulta insuficiente que los servicios sociales se aborden en una única asignatura del Grado, siendo necesario la incorporación de varias materias específicas -por ejemplo, Servicios Sociales I, II, III- como ocurre en otras titulaciones afines como Trabajo Social, con cuyos profesiones el educador/a social formará equipo en los contextos municipales y cuyo conocimiento del funcionamiento del sistema debe situarse al mismo nivel.

Por ende, se considera esencial la inclusión en los planes de estudio de una asignatura específica que prepare a los futuros profesionales de la Educación Social para desarrollar su labor profesional en los servicios sociales, desempeñando funciones que les son propias -de tipo individual/familiar, de carácter comunitario y de gestión de servicios- dentro de los equipos interdisciplinares que conforman las unidades de trabajo social (trabajadores sociales, psicólogos, etc.) y destacando la dimensión educativa en el marco de estas asignaturas (modelos educativos, metodologías de intervención, análisis de casos, etc.) como elemento clave para el ejercicio de la profesión con garantías de éxito, calidad y compromiso ético. Asimismo, se hace necesaria una mayor coordinación e interacción entre el mundo académico y la práctica profesional, a fin de poder articular un curriculum formativo fundamentado en el sustento teórico propio de la Pedagogía/Educación Social y en el conocimiento de la realidad a través de metodologías de carácter aplicado (realización de estudios de casos, elaboración de indagaciones de campo orientadas al análisis de aspectos concretos de los servicios sociales municipales, etc.).

\section{Referencias}

Asedes (2004). Definición de Educación Social. En CEESG (edit.) (2008), Documentos profesionalizadores (pp. 9-18). Santiago de Compostela: ASEDES, CEESG, CGCEES.

García Roca, J. (2006). Memorias silenciadas en la construcción de los servicios sociales. Cuadernos de trabajo social, 19, 197-212. 
Morán, M. C. \& Varela, L. (2017). Entrevista con... José Manuel Ramírez Navarro. Revista Galega de Educación, 67, 40-49.

Moyano, S. (2012). Acción educativa y funciones de los educadores sociales. Barcelona: UOC. 